\title{
Supplementary Results of the CAIS-1 Survey on Cheating in Undergraduate Engineering Programs in Saskatchewan
}

\author{
David M. Smith and Sean Maw \\ University of Saskatchewan \\ sean.maw@usask.ca
}

\begin{abstract}
In early 2016, engineering students and staff at the Universities of Saskatchewan and Regina were surveyed regarding their views and experiences as they relate to academic dishonesty. This paper summarizes some of the results from the gathered data.

Our first version of the Canadian Academic Integrity Survey (CAIS-1) was very similar to the Perceptions and Attitudes toward Cheating among Engineering Students (PACES-1) survey, as discussed in Carpenter et al [2]. With CAIS-1, a different set of demographic questions was posed along with some minor additions to the main bank of PACES-1 academic integrity questions, including three additional open-ended questions. The focus of this paper is on the results that came from the new questions as well as on those results that were not covered in the Carpenter paper although they did come from the original PACES-1 questions.
\end{abstract}

Certain demographics were found to be predictors of self-reported cheating frequency. There was a small but significant difference in cheating frequency based on gender, with males reporting cheating slightly more often than females. Academic average was found to negatively correlate with cheating frequency. The frequency of cheating in high school was a significant predictor for the frequency of cheating in university. These demographic results agreed with the results of PACES-1 and other research on academic integrity. One demographic result that did not agree with prior research was that cheating frequency did not increase with increased extracurricular involvement.

To better understand what influences engineering students to cheat, each respondent was given a score based on their self-reported frequency of cheating. This score was used to compare student responses in each of the following four categories: "situational cheating", "diffusion of responsibility", "personal responsibility", and "no choice but to cheat". The first three of these were used to analyze respondents to PACES-1 in Passow et al [11] and the construct "no choice to but to cheat" was added in the analysis of our CAIS-1 data. Situational cheating sub-scale scores were found to be a significant predictor of academic dishonesty. The other three were significant, but accounted for only a small portion of variance. In short, situations where a student judges the benefits of cheating to outweigh the risks are predictors of student cheating.

When asked if there was an acceptable time to cheat, most student respondents said that it was "never" OK to cheat. Despite this, many of these respondents reported engaging in cheating. Neutralizations were used to justify such behaviours, usually by putting the responsibility on instructors e.g. the workload forced us to cheat. For those who did say that there were acceptable times to cheat, a sizeable portion of the respondents said that they cheated to "help with their learning". Approximately 50\% of the students felt that faculty did not care about or were not engaged in preventing cheating behaviours. The students who held those views most strongly tended to care about cheating more than most other students.

Few statistical differences were found between students enrolled in ethics courses and those that were not. The differences that were found were similar to those between upper year and lower year students. These differences were confounded as the students in ethics courses were almost always upper year students.

Overall, the Canadians surveyed in this initial CAIS-1 study were similar to Americans surveyed over a decade ago. However, there were some notable differences and we found some new results that were not discussed in the earlier American studies.

Keywords: cheating, academic integrity, engineering, education

\section{INTRODUCTION}

In Smith et al [12], we reviewed the nature of academic dishonesty in North American (and primarily American) engineering programs, as it is currently understood in the literature. In [12], we noted that we had undertaken a parallel Canadian study utilizing the methods of Carpenter et al [2]. Our study employed a survey tool called CAIS-1 i.e. the Canadian Academic Integrity Survey (ver. 1), which was deployed at the Universities of Saskatchewan and Regina. It was quite similar to the Carpenter team's Perceptions and Attitudes toward Cheating among Engineering Students (PACES-1) survey tool [2]. We are currently in the process of submitting a manuscript which parallels the Carpenter et al paper, comparing and 
contrasting the CAIS-1 results with the PACES-1 results. However, there were some issues arising from PACES-1 that were not discussed in the Carpenter et al paper, and some of our additional demographic and research questions are not documented in the PACES-1/CAIS-1 comparison.

As such, this paper focuses on the results from CAIS-1 that are supplementary to those found in the Carpenter et al paper [2] and to our upcoming comparison of the PACES1 and CAIS-1 results. These involve demographic data not gathered in PACES-1, as well as new research questions introduced in CAIS-1. We also present a new way of looking at some of the PACES-1/CAIS-1 data.

We remind readers that cheating damages engineering schools in three fundamental ways. It short-circuits the achievement of learning objectives, it erodes the integrity of the evaluation system that is the basis for professional accreditation, and it demoralizes community members who do not cheat. As such, cheating (or academic dishonesty) is a serious issue.

This is the first detailed look at academic dishonesty specifically in undergraduate engineering programs in Canada, noting that all of the data is from Saskatchewan at this time.

\section{METHODS}

The Canadian Academic Integrity Survey was distributed to undergraduate students, graduate students, and faculty in the College of Engineering at the University of Saskatchewan (U of S) and the Faculty of Engineering and Applied Science at the University of Regina (U of R) during the Winter semester of 2016. Ethics clearance was provided by the U of S Behavioural Research Ethics Board and the U of R Research Ethics Board. An online version of the survey was hosted using FluidSurveys ${ }^{\mathrm{TM}}$. Email solicitations were sent to both engineering programs late in the winter of 2016 with links to the online survey. Paper copies of the surveys were also distributed to two classes, one at each institution. These classes included GE 449 Engineering in Society at the U of S, and ENGG 401 Engineering Law and Professionalism, at the $\mathrm{U}$ of $\mathrm{R}$. These classes were chosen so that a group of students currently being taught ethics and professionalism could be compared to the remaining undergraduate cohort. A total of 567 people responded to the survey, 515 of which were undergraduate students. This paper will only comment on the results of the undergraduate survey responses. The $\mathrm{U}$ of S students accounted for $65.1 \%$ of the undergraduate respondents and $\mathrm{U}$ of $\mathrm{R}$ students made up the remaining $34.9 \%$.

CAIS-1 was a derivation of the PACES-1 survey. PACES-1 was created by the Exploring Ethical decisionmaking in Engineering $\left(\mathrm{E}^{3}\right)$ research group that contributed a significant body of research towards understanding academic dishonesty amongst engineering students in the United States [2, 4, 6, 7, and 11]. The PACES-1 survey consisted of different question blocks, or sections, which focused on topics related to academic dishonesty. The sections of the PACES-1 survey can be summarized as the following:

1) Demographics,

2) Student perceptions and frequency of cheating,

3) Differences between types of cheating,

4) Common neutralizations,

5) Student/faculty understanding of policies,

6) Explanations for cheating,

7) Deterrents of academic dishonesty, and

8) Open-ended questions.

Several changes were made to PACES-1 to create CAIS-1. This was done with the permission of the $\mathrm{E}^{3}$ research group. Several demographic questions were added and removed in Part 1 to reduce the number to 20 demographic questions. The demographic questions were also moved to the front of the CAIS-1 survey. The questions that were removed were related to students in the United States and not to students in Saskatchewan i.e. questions related to sororities and fraternities. The wording was changed in one of the questions in Part 2 to better reflect modern cheating methods i.e. students now use cellphones and calculators rather than "PDAs". Additional questions were added to Parts 2, 3, and 7. The available responses in Part 5 were changed from a 3-point Likert scale in PACES-1 to a 5-point Likert scale in CAIS1 to better match other sections of the survey. CAIS-1's Part 8 section consisted of a completely new set of questions that allowed students to provide written opinions to open-ended questions.

\section{RESULTS}

As noted in the Introduction, our research team is currently in the process of submitting a paper that will directly compare parallel results from the PACES-1 and CAIS-1 surveys in a manner similar to the format presented in Carpenter et al [2]. To narrow the scope of this paper, we have chosen to explore the results of four areas that arose from the results of the CAIS-1 survey that will not be covered in a subsequent publication; these areas include demographic predictors of cheating frequency, student explanations of cheating, student responses to open-ended questions, and the effects of ethics course involvement.

\subsection{Demographic Predictors of Cheating Frequency}

CAIS-1 asked respondents 20 demographic questions in Part 1 of the survey. Historically, certain demographic factors have been found to predict cheating frequency in undergraduate students. To relate demographics to 
cheating frequency, scores were assigned to each student depending on their responses to 21 statements about cheating presented in Part 2 of CAIS-1. The survey respondents were asked to report how often they had performed an act of cheating at their current academic institution. A score was assigned to each possible response; a response of " 0 times" was given a score of 1 , a response of "1-2 times" was given a score of 2 , and a response of " $3+$ " was given a score of 3 . The severity of the cheating acts varied to not only cover obvious cheating acts, but also more controversial acts that may or may not be defined as academic dishonesty. Three items were excluded from the summative cheating score as the research team believed they did not necessarily constitute academic dishonesty. This means that a student could have a cheating score between 18 and 63. Most demographics showed no significant result when compared to frequency of cheating except gender, academic average, and high school cheating.

Gender is a common demographic factor to analyze in academic dishonesty research as differences between males and females have usually been present. In the CAIS1 study, there was a small but significant difference $(p<$ $.05)$ between the frequency of cheating in men $(\mathrm{avg}=23.1$, $\mathrm{SD}=5.0)$ versus women $(\mathrm{avg}=22.0, \mathrm{SD}=4.0)$ i.e. men reported cheating slightly more often than women. This trend agrees with other academic dishonesty research where men typically report cheating more often than women [1, 9, and 10] as well as with the results of PACES1 [11]. Of the undergraduate respondents to CAIS-1, $74.2 \%$ were male and $25.6 \%$ were female.

Academic dishonesty was found to have a significant negative correlation with academic average $(p=001)$. As student averages increased, they were less likely to selfreport academic dishonesty. This was the expected result as a lower academic average is often found to be a factor in the frequency of academic dishonesty self-reporting [1, $5,9,10$, and 11].

Our team was interested in knowing if high school cheating behaviour would differ between Canadian and United States schools. From the results of CAIS-1, it was found that high school cheating frequency significantly predicted the frequency of self-reported cheating of engineering students $(p<.005)$. A similar result was found by the PACES-1 researchers [6]. Interestingly, most undergraduate respondents to the CAIS-1 survey (68.9\%) reported having never cheated in high school. This was surprising as most respondents to PACES-1 (60.2\%) reported some cheating during their time in high school [2]. This large difference in the reporting of cheating amongst high school students in two different countries at first seemed quite significant. However, one reason for this difference may be the difference in location of the demographic section in each survey. CAIS-1 asked about high school cheating before asking students to evaluate different cheating acts. PACES-1 asked about high school cheating at the very end of the survey. It is possible that after reading the acts presented in PACES-1, many students may have re-evaluated their original definition of academic dishonesty and may have been more critical in their self-evaluation of high school cheating. Either way, it seems that cheating in high school remains a predictor of academic dishonesty in Saskatchewan universities. More studies at universities across Canada could reveal if this is a country-wide characteristic.

One Canadian demographic result of interest that was not correlated to cheating frequency was extracurricular involvement. In US studies, increased involvement in sports and clubs is frequently linked to higher levels of selfreported academic dishonesty [1, 5, 10, and 11]. Most surveyed students in CAIS-1 reported being involved in some sort of extracurricular activity (56.9\%). Surprisingly, the CAIS-1 data revealed no significant difference between students who do participate in extracurricular activities and those who do not when it came to frequency of cheating ( $p$ $=.740$ ). Extending CAIS-1 to more academic institutions could reveal whether this is an anomaly or whether it is typical of engineering institutions across Canada.

\subsection{Student Reasons for Academic Dishonesty}

Another block of the survey questions asked students to provide explanations for cheating and to reveal who they thought was responsible for academic dishonesty. First, scores were assigned to students based on their selfreported frequency of cheating as explained earlier. To analyze student explanations for cheating, four sub-scales were developed, based on a block of questions within CAIS-1. Three of these sub-scales were adapted from analyses performed by Passow et al [11] when analyzing the same questions in PACES-1.

The three sub-scales that were borrowed from the analyses in Passow et al [11] included i) situational cheating, ii) diffusion of responsibility of cheating to external sources, and iii) personal responsibility for cheating. The first sub-scale, situational cheating, was assessed through responses to four statements i.e. I would cheat to avoid getting a poor or failing grade in a class; I would cheat if doing so helped me retain my financial assistance; I would cheat in a class if it seemed that everyone else was cheating; and I would cheat to avoid letting my family down if I failed. Situational cheating can be described as the "predicted decision to cheat in situations when the benefits outweigh the costs" [11]. The second sub-scale, diffusion of responsibility of cheating to external sources, indicated how likely a student would agree with the following two statements: It is the institution's responsibility to prevent cheating, and It is the instructor's responsibility to prevent cheating. The final sub-scale adopted from Passow et al [11] was personal responsibility for cheating. This included three survey statements i.e. It is my responsibility to prevent cheating; If 
I saw another student cheating I would report the student to the instructor; and If I saw another student cheating I would confront the student. A fourth sub-scale, no choice but to cheat, was added for the analysis of CAIS-1 and was assessed using two statements: Cheating is a necessary part of life and I have to cheat just to get grades good enough to compete with other students at this school. This sub-scale was used to measure a student's feeling that the present situation within their program necessitated academic dishonesty just to get by.

Using the frequency of cheating score assigned to each respondent, it was found that all four sub-scales were significantly correlated with cheating behaviours.

- $\quad$ Situational: $\mathrm{r}=.50, \mathrm{p}<.001$,

- Diffusion of responsibility: $r=-.10 \mathrm{p}=.032$,

- Personal responsibility: $\mathrm{r}=-.14, \mathrm{p}=.004$,

- No choice but to cheat: $r=.396, p<.001$

A hierarchical regression was applied to the four sub-scales with situational cheating in the first block, followed by no choice but to cheat, diffusion of responsibility to external sources, and personal responsibility for cheating. Situational cheating accounted for a significant proportion of variance in the frequency of cheating $\left(\mathrm{R}^{2}=.249, p<\right.$ $.001)$. None of the remaining three subscales significantly accounted for any additional variance.

The researchers behind PACES-1 found that situational cheating sub-scale scores were a strong predictor of cheating, specifically on homework [11]. Analysis of the CAIS-1 results demonstrated situational cheating scores to be a major predictor of the frequency of general academic dishonesty. However, the analyses of the CAIS-1 data did not differentiate between homework and exam cheating, whereas the analyses of the PACES-1 did do this. Although strongly correlated with cheating frequency, the three remaining factors play a lesser role in predicting frequency of cheating. The inclusion of the fourth subscale, no choice but to cheat, is important since a culture of cheating is often assumed to exist amongst engineering students. If such a culture exists, it would be expected that a student might agree that the only way to survive within their engineering program would be to engage in academic dishonesty frequently. However, results of a hierarchical regression show that this explanation only weakly translates to a higher frequency of cheating, at least in Saskatchewan. When "no choice but to cheat" was added to the model, its addition did not significantly account for any more variance than the situational explanations $\left(R_{\text {change }}^{2}=.005\right)$.

\subsection{Student Responses to Open-Ended Questions}

An additional section (Part 8) was added to the CAIS-1 survey that was not in the original PACES-1 survey. This section gave students a chance to provide a written response to the survey and to communicate to what extent they cared about cheating in their engineering program. Written answers were coded by grouping similar types of answers into categories that were determined organically during this text analysis stage. Numbers of responses in the emergent categories were then tallied. If a respondent gave multiple answers within their response, two or more code numbers were assigned to their answer. The first question, "when is it OK to cheat", had a response rate of $75.9 \%$ for the undergraduate respondents. The majority $(66.2 \%)$ responded "never".

The two most popular responses for when it is OK to cheat included "group work" and "on assignments" at $6.6 \%$ and $7.4 \%$, respectively. If a student was to choose a time to cheat, it seems situations involving group work and homework assignments are the most common. The question "if there is an $\mathrm{OK}$ time to cheat, why is it OK to cheat then?" had a response rate of $25.6 \%$. Most students did not answer this question as they had already responded "never" to the previous question. Answers to question two categorized as "never" were removed for analysis, leaving 128 undergraduate respondents to this question. A very interesting finding is that $24.2 \%$ of these respondents reported that "cheating will help with my learning". This is an issue not explored in other sections of the survey. It reveals an important positively motivated neutralization for students to cheat on homework. Using solution manuals could become easily justifiable if a student believed their use was necessary for learning class material. Other popular reasons given were that "the instructor has done a poor job" (11.7\%) and that "assignments are acceptable to cheat on" (12.5\%).

The third question in Part 8 was broader than the first two and asked respondents to "describe the general attitude towards cheating in this College/Faculty, as you see it". This question provided respondents an open forum to relate their feelings on cheating in their engineering school. It had a response rate of $66.6 \%$ of undergraduate students. Since the answers given were very broad, answers were assigned to one of four broad categories: negative attitude, neutral attitude, positive attitude, or I don't know. A negative attitude means that the respondent generally felt that the cheating was a problem in the school. Typical responses included "the faculty are apathetic", "cheating is a major problem", "harsher punishments are needed", or "cheating is necessary". The percentage of students falling into this negative attitude category was $46.9 \%$. Students put into the "neutral attitude" category generally reported that cheating was a problem, but that the instructors and institution were working to solve it. Typical answers included "cheating is frowned upon, but it still happens", "there is a difference between cheating on assignments and exams", "the definition of cheating is too unclear", or "cheating using solution manuals is OK" such that $22.4 \%$ of undergraduates were placed in this category. Respondents who had a positive attitude generally believed 
that the school was actively trying to stop cheating or felt that cheating was unacceptable $(27.4 \%$ of undergraduates who responded, were placed in this category). The final category, "I don't know", included respondents who responded that they were unsure of the attitude in their college/faculty. This accounted for $3.2 \%$ of undergraduate students who responded.

The fourth and final question in Part 8 asked students "to what extent do you care about cheating in your engineering program?" This question had a response rate of $67.2 \%$ among undergraduates. The most common response was "somewhat" at $48.3 \%$ followed by "a lot" at $34.7 \%$. It seems that cheating is not a huge issue for most engineering students, but interesting results arise when comparing responses to questions 3 and 4 from Part 8 . These results are displayed in Figure 1, below. Each bar colour represents a different student attitude as seen in question 3, and this is compared to each possible response for question 4 regarding how much they care about academic dishonesty. Those students who were placed in the "negative attitude" category responded "enormously" more often than both other categories combined. If the categories "a lot" and "enormously" are combined, respondents in the "negative attitude" category have the highest response at $49.4 \%$ followed by "positive attitude" at $42.6 \%$. Students who are frustrated with cheating at their school, or at least think that it is a problem, are those who care the most about addressing cheating. Note that there are limitations to this analysis due to the subjective categorization method. However, further versions of the survey could provide more insight into this finding and a better understanding of the natural categories that arise from it.

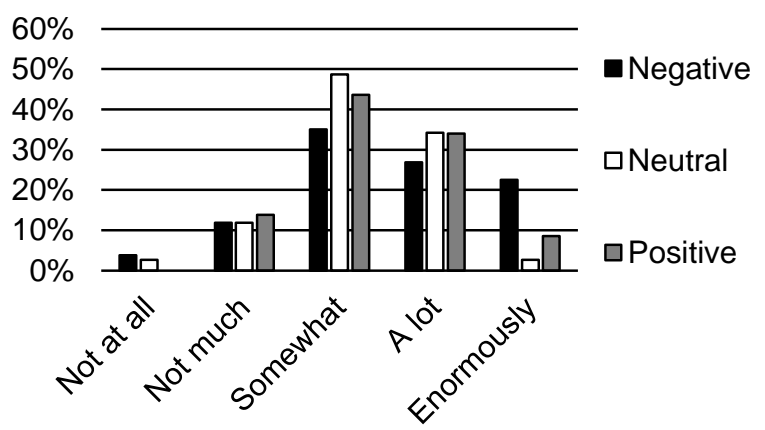

Fig. 1. A comparison of the answers to Question 3 (bar colours) and Question 4 (x-axis categories) from Part 8.

\subsection{Effect of Ethics Course Enrollment}

Engineering students across Canada are required to take training in ethics and professionalism during their academic career [3]. Both engineering programs surveyed using CAIS-1 specifically included students currently enrolled in an ethics and professionalism course. These courses are offered later in student academic careers and are usually taken in their fourth or fifth year. Since students are receiving training in ethics and professionalism, the authors believed that these students would be more likely to identify cheating acts as academic dishonesty more often than students who had not taken the same training. The goal of analyzing this data was to identify whether these ethics course were having a tangible (positive) effect on the ethics training of engineering students.

With the permission of the instructors of these ethics courses, students were approached during their class time and were asked to participate in the CAIS-1 survey. Students were provided with either a paper copy of the survey or a link to the survey online. A total of 181 students in these courses responded with $56 \%$ of the respondents attending the $\mathrm{U}$ of $\mathrm{S}$ and $44 \%$ attending the $\mathrm{U}$ of R. Initial comparisons showed little difference between the perceptions of cheating acts of students enrolled in an ethics course and those who were not enrolled. The largest perception difference between these groups was found with item 2 in Part 2 i.e. permitting another student to look at your answer during a quiz or exam. Approximately $69 \%$ of the ethics course students said that this act was cheating versus $78 \%$ of the non-ethics course students. While distressing, this difference would be more noteworthy if large differences were seen in more items, but this trend was not observed.

More distinct differences were present when comparing the frequency of cheating between the two student groups. A total of $72 \%$ of ethics course students reported never performing item 1 in Part 2 (copying from another student during a test or quiz) and $74 \%$ responded having never performed item 2 (permitting another student to look at your answer during a quiz or exam). Compare this to the responses of students not in an ethics course where $87 \%$ responded never to item 1 and $99 \%$ to item 2. Similarly, for six of the other cheating acts presented to students, there was at least a 10\% decrease in responses of "never" of the ethics course students compared to students not in an ethics course. If the scoring system described earlier for "frequency of cheating" is used, the mean cheating frequency score for students in ethics courses was 22.4 (SD $=5.95)$ and the mean score for non-ethics course students was 22.0 ( $\mathrm{SD}=5.10)$. In other words, the students enrolled in an ethics course reported a higher frequency of cheating, although the difference was very small.

Initially, this may seem to be a discouraging result. However, as noted earlier, students enrolled in ethics courses are typically in the later years of their undergraduate career. Analyzing the responses of students enrolled in their fourth year or higher revealed similar cheating frequency and perception patterns. For the eight items that demonstrated a decrease of $10 \%$ between ethics students reporting "never" versus students not in ethics courses, five of those cheating items showed the same trend when comparing students in their fourth year or higher to 
students in their first, second, or third year. When comparing cheating frequency scores, students in their fourth year or higher had a mean cheating score of 22.5 (SD $=5.86$ ) and students in either first, second, or third year had a mean score of $22.1(\mathrm{SD}=4.85)$.

Some distinct differences did exist between year of study and ethics course enrollment i.e. a few items showed large differences in opinion between fourth year and higher students and lower years that were not seen in the ethics course results. However, similar results blurred any distinct trends and made it difficult to distinguish if differences were a result of students having been educated in ethics and professionalism or if their years of enrollment in engineering had encouraged certain behaviours.

\section{CONCLUSIONS AND DISCUSSION}

Most of the results from the analyses of the demographics of the survey responses agree with other research. Male engineering students are typically found to self-report that they cheat more often than female students, and our survey found the same result. This is not to say that the male students cheat more often because they are male. Rather, it could be a function of the disproportionate numbers of male and female students enrolled in engineering. If more female students were enrolled, the amount of cheating in both genders could converge, for example. Researchers have seen this convergence of behaviour in some programs already [8].

Academic average and high school cheating behaviour followed the expected trends as well. The higher the academic average of the student, the less likely they were to engage in academic dishonesty. If a student reported cheating in high school, they were more likely to cheat in university. To gain a better understanding of Canadian engineering students, our research team would like to analyze demographic trends at more institutions across Canada to see if it is consistent or if results differ from region to region.

There are many explanations for student cheating. Since factors and variables are diverse, it can be difficult to pinpoint exact reasons and influencers on student behaviour. The student explanations presented in this paper provide an initial glimpse of student reasoning. Our results agreed with the results from PACES-1 i.e. that situational cheating is a major indicator of whether a student will choose to cheat or not. It is common for engineering students to be overwhelmed with high workloads. Situational cheating occurs when students find a way to alleviate stress through academic dishonesty and the benefits seem to outweigh the risks. However, there are limitations to this result. The questions that make up situational cheating in this survey are rather limited. There are more situations that would provide opportunities for students to alleviate stress which could be better represented in a revised survey. The addition of the category, "no choice but to cheat", expands on the work of PACES-1 and provides insight into engineering student culture. Although students may choose to alleviate stress through academic dishonesty, it seems that most students do not feel that academic dishonesty is required to get by. Academic dishonesty may be lessened by reducing student workloads and by removing the competition for grades within engineering.

The CAIS-1 results were enhanced by giving students open-ended questions through which they could express their opinions and frustrations with academic dishonesty. Despite most students expressing that it is never $\mathrm{OK}$ to cheat, the frequency of cheating is still unnervingly high. Researchers typically explain this discrepancy as a function of neutralizations [5]. Many of the commonly cited neutralizations were found when students were asked as to when it was OK to cheat. These included blaming instructors, pointing to social norms, and criticizing high workloads. One interesting neutralization that came out of our analysis was that some students feel cheating will help them learn the material better. Some engineering students rely on solution manuals and websites that provide homework solutions such as Chegg and Course Hero to aid them in their work [13]. Perhaps instructors can do better at finding new methods that aid students in their effort to understand the material that will remove their reliance on secondary cheating sources. Of course, dramatic changes in evaluation could also strongly influence behaviours associated with academic dishonesty.

The final focus of interest in this paper was the effect of ethics course enrollment. Unfortunately, no conclusive results could be determined from CAIS-1. Other studies by the $\mathrm{E}^{3}$ team have sought to explore ethical education in more depth [4 and 6]. Although analyzing ethics courses at other universities could provide more information, a separate, dedicated survey may provide more information on the effectiveness of ethics education within Canadian engineering programs.

\section{FUTURE WORK}

An improved and revised CAIS-1b is now ready for deployment, and we are looking for Canadian engineering programs that would like to take part in this expanded study during the Fall of 2017.

As well, we are preparing to deploy CAIS-2 in Saskatchewan, which will focus on more practical and specific issues around cheating in engineering programs. Specifically, we will be focusing on "ways and means" as well as deterrents. We anticipate piloting the CAIS-2 survey tool at $\mathrm{U}$ of $\mathrm{S}$ and $\mathrm{U}$ of $\mathrm{R}$ in 2017/18.

\section{Acknowledgements}

The authors would like to thank Don Listwin, funder of the Huff Chair at the University of Saskatchewan, for his 
vital support. Huff Chair research funding made this work possible. We would also like to thank Dr. Trevor Harding of California Polytechnic and the $\mathrm{E}^{3}$ team for letting us use their Perceptions and Attitudes toward Cheating among Engineering Students (PACES-1) test battery, and for sharing sage advice. As well, we appreciate the support of Douglas Wagner, Susan Bens, Sarah Sangster, the Engineering Student Centre (Saskatchewan), the Dean's Office (Regina), and the instructors of GE 449 and ENGG 401 for their help in distributing the survey and in analyzing results.

\section{References}

[1] William Joseph Bowers, Student Dishonesty and its Control in College. New York, NY: Bureau of Applied Social Research, Columbia University, 1964, 399 pp.

[2] Donald D. Carpenter, Trevor S. Harding, Cynthia J. Finelli, Susan M. Montgomery, and Honor J. Passow, "Engineering students' perceptions of and attitudes towards cheating," Journal of Engineering Education, vol. 95, no. 3, pp. 181-194, July 2006.

[3] Engineers Canada. 2016 Accreditation Criteria and Procedures. Engineers Canada. Available as of May 33, 2017 from https://engineerscanada.ca/sites/default/files/accredit ation-criteria-procedures-2016-final.pdf

[4] Cynthia J. Finelli, Matthew A. Holsapple, Eunjong Ra, Rob M. Bielby, Brian A. Burt, Donald D. Carpenter, Trevor S. Harding, and Janel A. Sutkus, "An assessment of engineering students' curricular and co-curricular experiences and their ethical development," Journal of Engineering Education, vol. 101, no. 3, pp. 469-494, July 2012.

[5] Valerie J. Haines, George M. Diekhoff, Emily E. LaBeff, and Robert E. Clark, "College cheating: immaturity, lack of commitment, and the neutralizing attitude," Research in Higher Education, vol. 25, no. 4, pp. 342-354, December 1986.
[6] Trevor S. Harding, Donald D. Carpenter, and Cynthia J. Finelli, "An exploratory investigation of the ethical behavior of engineering undergraduates," Journal of Engineering Education, vol. 101, no. 2, pp. 346-374, April 2012.

[7] Matthew A. Holsapple, Donald D. Carpenter, Janel A. Sutkus, Cynthia J. Finelli, and Trevor S. Harding, "Framing faculty and student discrepancies in engineering ethics education delivery," Journal of Engineering Education, vol. 101, no. 2, pp. 169-186, April 2012.

[8] Rozzet Jurdi, H. Same Hage, and Henry P. H. Chow, "Academic dishonesty in the Canadian classroom: behaviours of a sample of university students," Canadian Journal of Higher Education, vol. 41, no. 3, pp. 1-35, 2011.

[9] James S. Leming, "Cheating behavior, subject variables, and components of the internal-external scale under high and low risk conditions," Journal of Educational Research, vol. 74, no. 2, pp. 83-87, 1980.

[10] Donald L. McCabe, and Linka Klebe Trevino, "Individual and contextual influences on academic dishonesty: a multicampus investigation," Research in Higher Education, vol. 38, no. 3, pp. 379-396, June 1997.

[11] Honor J. Passow, Matthew J. Mayhew, Cynthia J. Finelli, Trevor S. Harding, and Donald D. Carpenter, "Factors influencing engineering students' decisions to cheat by type of assessment," Research in Higher Education, vol. 47, no. 6, pp. 643-684, September 2006.

[12] David M. Smith, Susan Bens, Douglas Wagner, and Sean Maw, "A Literature Review on the Culture of Cheating in Undergraduate Engineering Programs," in Proc. Canadian Engineering Education Association Conf., CEEA 2016, (Halifax, NS; 19-22 June 2016), 1 pp., 2016.

[13] Brad Wolverton, "The New Cheating Economy," The Chronicle of Higher Education, vol. 63, no. 1, 2016. 